\title{
Individualized Episteme for Multi-Agent Situations in Virtual World
}

\author{
Jong-Hee Park ${ }^{1}$, Jun-Seong Choi ${ }^{2}$ \\ ${ }^{1}$ Professor, School of Electronics engineering, Kyungpook National University, Republic of Korea. \\ ${ }^{2}$ Ph.D., Student, School of Electronics engineering, Kyungpook National University, Republic of Korea.
}

ORCID: 0000-0002-3165-1549 (Jong-Hee Park)

\begin{abstract}
Virtual world-based education systems pursue to provide realistic pedagogical experiences in realistic situations. The diversity and realism of situations simulated in the virtual world determines the quality of pedagogical experience in immersed learning. A situation grows more complicated as it involves more agents, who belong to the same multi-agent event or many independent events. Unlike most of the serious games and conventional narrative systems, we indiscriminately model those agents to be autonomous humans regardless of their roles. That is, they have their own beliefs enabling them to behave autonomously and independently from each other. This epistemic differentiation itself provides a major source of additional situation variability compared to the omniscientview or protagonist-centered situations of those conventional narrative systems. Each agent is designed to be incessantly update their respective episteme according to ever-changing background-world conditions to adapt their behavior proactively and reactively. Such episteme cannot be perfect due to incomplete information and partial observation, leading to misconception and epistemic discrepancies, between fact and belief and inter-agent, further deepening the situation variability. With epistemic multiplicity for autonomous and independent agents our simulation method affords the highest diversity and fidelity of pedagogical situations involving multiple agents. We developed a dynamic event-coupling scheme based on schematic functions other than the conventional pre-authored planning. This scheme can not only maximize the situation variability but drastically alleviate the authoring cost along with a real-time animation method compared to monolithically-authored conventional simulation systems.
\end{abstract}

Keywords: Immersive pedagogical experience, Multi-agent situation, Virtual world simulation, Individualized episteme,

\section{INTRODUCTION}

Virtual world-based education systems pursue to provide realistic pedagogical experiences immersed in diverse situations [1-2]. The variability and realism of situations simulated in the virtual world determines the quality of pedagogical experience in immersed learning [3-4]. The omniscient or protagonist-centered view in conventional narrative systems like Interactive Storytelling (IS) inevitably results in deterministic development of story, making it infeasible to react to unforeseen events or to enhance story variability [2]. This inherent limit renders them inadequate for many types of education systems demanding variability over coherence of story. As a measure to alleviate this limit, our simulation first decouples agent's belief from the reality to reflect the limited epistemic constraints of humans.

A realistic situation in general comprises many concurring events, pre-planned or unforeseen, each of which in turn usually involves multiple agents. Unlike most of the serious games and conventional narrative systems, we indiscriminately model those agents to be autonomous humans regardless of their roles. That is, they have their own beliefs enabling them to behave autonomously and independently from each other. All the unique judgement of humans and affective reaction premises their own episteme. This epistemic differentiation itself provides a major source of additional situation variability on top of the variability due to the inherent complexity of intentional events often involving many agents. This breakaway from the omniscient perspective in conventional simulation systems greatly enhances the realism in the simulation of pedagogical situations. Each agent in our simulation is designed to incessantly update their respective episteme according to ever-changing background-world conditions to adapt their behavior proactively and reactively [5]. Such episteme cannot be perfect due to incomplete information and partial observability, leading to misconception and epistemic discrepancies between fact and belief and inter-agent, further deepening the situation variability. With epistemic multiplicity for autonomous and independent agents our simulation method affords the highest diversity and fidelity of pedagogical situations involving multiple agents [5]. The resulting epistemic discrepancy and imperfect episteme can cause numerous problems such as 'unconsciously', 'delusion', 'unforeseen event', 'misplaced belongings', 'negligence', 'hidden camera', 'mutual misunderstanding', etc. These problems may lead to erroneous judgements and subsequently emergent situations, which the conventional narrative systems struggle to be able to generate [3].

In a sharp break with the conventional view that (part of) the background world is passive part of each situation or scene, the situations and its associated (part of) background world are decoupled from each other. We devise a dynamic eventcoupling scheme based on schematic functions other than the conventional pre-authored planning. This scheme can not only maximize the situation variability but drastically alleviate the authoring cost, which would otherwise be prohibitively high with its associated monolithic authoring of every possible path of storylines. Within dynamic event coupling, the coupling intimacy and the resulting expressivity and coherency of situations vary widely according to the granularity of coupling $[6,7]$. Our model selects the actions of agents as the primitive units of coupling, which enables events to be intimately 
connected via actions (e.g., walk, eat) of the agents cast in their roles as well as via inter-event association rules. All the events (and agents) are instantiated onto a global timeline to be situated in historical contexts instead of being contextualized in relative timing only among interacting characters within each scene [6]. To realize this scheme, the parameters of functions are to be separated from situations and situations are to be independent from each other so they can be indirectly linked via a background world. This full-blown background world provides a comprehensive spatio-temporal stage for independent events to become meaningfully coupled with each other [5]. These events are coupled only if they are interrelated according to the association rules and conditions from their common background world [5].

Individualized episteme and dynamic event coupling collectively affords very high variability of simulated situations involving many concurring multi-agent events. Supported by a real-time animation scheme [7], our cyber-world system still is scalable enough to underlie practical simulation systems. We demonstrate the feasibility of our simulation method with an implementation for typical multi-agent situations.

\section{EPISTEMOLOGY DECOUPLING AGENTS' BELIEFS FROM REALITY}

Before the cosmos was inhabited by humans, the reality comprises only the natural (or physical) world. They form a community, whose associated social world is built mostly on agreements among its member humans. The reality in our cyber cosmos consists of the physical world and the social world thereon. In fact humans act on their beliefs instead of the reality. The community knowledge (pieces) can affect its member human only when they become part of her belief through assimilation process. Overall, the reality corresponds to ontology while belief to epistemology. The indifferentiation between the reality and belief, as is the case in conventional systems [3, 8, 9], would leave a major portion of situations happening in our Cosmos unaccounted for. NPCs in conventional narrative systems have still not been designed to affect changes of story flows, weakening sustained immersion of their users [10]. This limitation can be methodically resolved with individualized episteme.

To elaborate its complex architecture, the overall virtual world (or Cosmos) as the base of our simulated situations is modeled in terms of (primitive cosmic units of) entities and their interrelationships [5]. The Cosmos is layered in terms of the Reality, which in turn is composed of the Physical and Social worlds, and, on top of it, the Conceptual Worlds of its inhabitants or agents. Cosmos $\left\langle R(\mathrm{t}),\left\{C_{i}(\mathrm{t})\right\}\right\rangle, R=\langle P, L\rangle$ where $R, P$ and $L$ each denote Reality, Physical World and Social World with $<>$ denoting 'comprises'; $i=1,2,3, \ldots$, \# of agents; Agent i's Conceptual World $C_{i}(\mathrm{t})=\left\langle M_{i}, E_{i}\right\rangle$ where $M$ and $E$ denote its Mind and Episteme, respectively [11]. Technically $C_{i}(\mathrm{t})=f_{i}(R(\mathrm{t}))$ where $f_{i}()$ is the mapping function of agent ${ }_{i}$. That is, $C_{i}(\mathrm{t})$ maps $R(\mathrm{t})$ only partially and often erroneously. Each community member maps on her belief other members' beliefs as well, that is, $E_{i} \supset f_{i}\left(E_{j}\right), \mathrm{j} \neq \mathrm{i}$. This reciprocity between agents' beliefs can bring on a (theoretically) infinite mirroring in epistemology. This mirroring becomes evident, for instance, in people trying to outsmart their opponent when playing the rock-paper-scissors game.

Episteme in an overall perspective encompasses recognition as well as belief. The mapping from the reality to the episteme in the recognition process generates different versions of the same entity. For these versions, we have to deal with numerous issues such as inter-layer linking and discrepancies. Human's episteme can never be perfect, i.e., bound to be partial vertically (or not elaborate the reality enough) and horizontally (or not cover the entire range of the reality) and distorted during the mapping. Further, those discrepancies happen not only between the layers but between the beliefs of fellow agents as illustrated in Fig. 1. Their respective episteme is indicated by a red rectangle (with its id $\mathrm{H \# )}$ over the common Reality. The detailed notation about the Reality is beyond our scope [6]. Different epistemes overlap with each other. They manifest themselves in the forms of 'actual vs expected/planned/forecast', 'truth vs beliefs', 'different memories of their common childhood', etc.
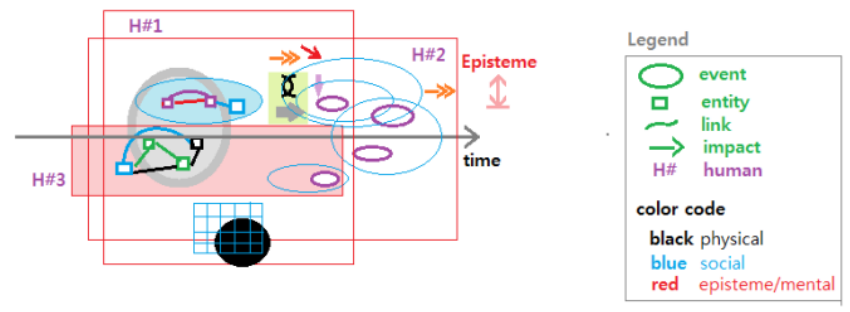

Fig. 1. Respective beliefs of fellow human agents

Among a myriad of typical situations involving epistemic discrepancies are: 'I believe she did it unconsciously.' 'When did she realize it had been lost?' 'He misplaced it.' 'They investigated to find out.' 'We have to rehearse once more before the show.' 'He threats with a broken nose.' 'Is it by negligence or premeditated?' 'He is the only member to experience it first-hand.' 'They could not agree on what had happened.' 'There are only allegations but no smoking gun to prove the truth.' 'We pretended to be unaware of.' An intricate instance of epistemic discrepancy should be associated with a hidden-camera TV program, where all the participants would have colluded on the scheme hidden from the victim only while the entire scheme has been explained to its (large) audience beforehand. Such a victim would behave differently if she knew her every move being watched.

The reality is captured onto the episteme through direct sensation or indirect experience. Any experience can be gained only via the physical world so diverse physical aspects eventually affect the contents of the resulting episteme. On the other hand, human's memory is bound to diminish or be outdated over time, thereby leading agents to make erroneous judgements. Conversely these limits of the memory could be a bliss for our simulation in that it paves a way to simplifying our human behavior model to a quasi-memoryless system. Such a memoryless system could simulate the episteme that its agent refers to be confined to just as far as the agent remembers. Of a community, conventional wisdom or commonsense knowledge could be modeled as statistical averages over its member 
humans' knowledge. Those average pieces of knowledge would be used by the agents as the practical references to anticipate behaviors typical or expected of fellow agents.

\section{DYNAMIC EVENT COUPLING METHOD AND REAL-TIME ANIMATION TECHNIQUE}

While every event in a conventional narrative system is anticipated in the authoring time (with a pre-contrived nondeterminism in a limited scope $[8,12]$ ), our dynamic event planning allows events, preplanned and unforeseen alike, to be coupled in the execution time. This planning scheme eliminates multiplicative explosion of possible situation flows inevitable in monolithic authoring of story comprising a sequence of events. One of its key requirements is a complete set of schematic events (formulated in a reusable form) which can be instantiated according to the varying conditions of the background world. Consider an example situation involving a theft event and entailing events of report and police chase as illustrated in Fig. 2. Our model provides only instances of agents such as a thief, victim, policeman, etc. equipped with actions and several schematic events. These schematic events are instantiated with their associated background world conditions and accordingly coupled into a progression of a situation [13]. Those coupling are not just due to the physical or social interaction but also epistemic implications. In a police check to track a crime suspect at large, for example, an innocent passerby could be mistaken for the suspect. This misidentification would be due to the policeman's epistemic error that the victim's looks match the suspect's description, which is not true in the reality.

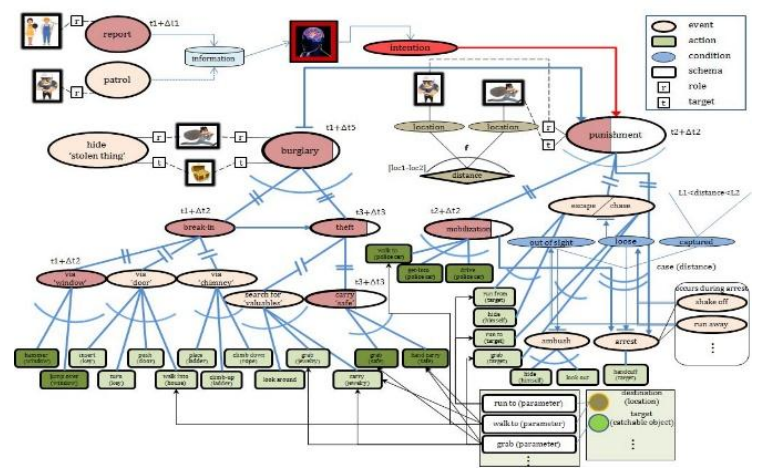

Fig. 2. High situation variability realizable from dynamic event-coupling scheme

This event involves many subevents in layers as illustrated in Fig. 2, which are interconnected in diverse manners, e.g., conjunctively, alternatively, etc. Due to our dynamic event coupling scheme (indicated by double dashed blue links), every story flow need not (actually, cannot) be predicted beforehand unlike in conventional monolithic authoring. As a result, the authoring burden grows only arithmetically instead of exponentially with the number of subevents and their associated factors. Notice this planning-stage advantage in authoring burden could be significantly widened taking into effect the fact the plot is to be elaborated with respect to interevent timing and real-time conditions of the background world.
A complicated situation usually depends on its preceding situations, which could be recursively repeated endlessly at least theoretically. Such inter-event dependency would pose a prohibitive burden on single-plot generation or monolithic authoring [8]. On top of this burden, individualized episteme would further aggravate the authoring scalability. This could apply even to our simulation model though it is based on the dynamic event coupling instead of pre-planned authoring. Considering the humans' memory is bound to diminish over time, however, their decision-making process could be modeled as a quasi-memoryless system. That is, the reverberating effects via agents' episteme due to inter-situation dependency would subside within limited time spans from the current situation the agents are now involved in.

Implementation of this dynamic coupling demands both realtime performance and animation flexibility to support the action-level coupling. This performance and flexibility is realized by the agent modeling based on multiple priority queues each corresponding to an autonomous agent's planned actions [13]. The actions, the primitive units of coupling, belong to capability of an agent and is the animated unit of implementation. This fine level of animation unit leads to an animation technique to enable intra-agent coupling of events beyond conventional inter-agent coupling. This parallel-action animation technique integrates into a composite action parallel actions of one agent playing roles in different events (e.g. phoning to invite a guest and walking to buy grocery) as judged possible by kinetic constraints on that agent [7]. This parallelism affords animation scalability against the infinite variability of changes often occurring in complicated multievent situations the virtual world. It also enables new events to be efficiently executed in reaction to abrupt changes in relevant conditions. This reusable action level animation is complemented with limited recognition capability of agents to account for numerous situations due to their misconception. Further, events in our planning are aligned along a global timeline in the spatio-temporal space so as to be scheduled according to their exact timing beyond relative precedence between events, which adds yet another non-determinism to our planning.

\section{PLAN EXECUTION MECHANISM FOR MULTI- AGENT SITUATIONS}

In a multi-agent situation, many concurrent plans associated with their corresponding events are to be executed correctly despite of any inter-event interweaving. We developed a plan execution mechanism based on priority queues [5]. Those plans in general are hosted by independent agents. All identified actions in new plans are interleaved in between the existing actions of the priority queues corresponding to their associated initial plans. In our level-wise execution, firstly pre-planned or unforeseen events alike are ordered according to their interevent priority and observed in a sequential or an interleaved manner. The actions belonging to each event are arranged according to their intra-event priority and their precedence is strictly maintained across their respective queues. Each queue set hosted by the same agent corresponds to an agent with its own episteme. The motion level allows an action to be flexible 
for reflecting any impact from the background-world conditions [7] unlike the conventional monolithically-authored animation $[8,12]$. The outcome expected with each intra-event plan is guaranteed to be reality correct unless interrupted by unforeseen events or disruptive conditions. Conversely, those events whose preconditions are not currently satisfied are set to disabled. As a result, independent events can arbitrarily be interleaved according to their priority enabling coincidental coupling to coherently occur among those events.

\section{EXAMPLES AND IMPLEMENTATION}

Each of our simulated human agents has its respective episteme instead of one uniform omniscient perspective on the world. Such an agent is designed to be with its individual recognition capability as the feeder to its own belief. An agent's episteme in practice suffers from diverse problems due to incomplete recognition and inferencing capability, which still is conducive to realizing epistemic individuality of agents. To react to timevarying situations, an agent is designed to use its ability to recognize not only primitive attributes of entities but the actions (the finest comprehensive semantic units) and events. Agents would incessantly update their beliefs according to everchanging background-world conditions to accordingly adapt their behavior. Notice every phase of their recognition process is subject to errors due to darkness, blocked view, a tiny, transparent or distant object, blurry eyesight, etc. Such misconception and epistemic discrepancy could entail subsequent erroneous judgements. This discrepancy between the up-to-second world conditions and the agent's episteme further elaborates situation variability. The recognition timing as a part of dynamic coupling also could significantly affect the way the situation develops.

We demonstrate how epistemic discrepancies diversify the situations involving one or more agents, which would otherwise be straightforward or strictly dictated by various laws. Those discrepancies can occur between reality and episteme or between different agents' episteme. An example situation comprises independent events, each of which is composed of the animated unit of agents' actions. Each action in turn is constructed by a small set of reusable primitive motions [7]. Consequently, an intricate situation is shown to be implemented in terms of a small set of primitive motions.

Fig. 3 shows a short sequence of motions to constitute 'walk' to a destination. A monotonous progression of the walking event could be disrupted anytime, and our real-time animation scheme accommodate any abrupt change based on action- or motion-level assembly technique [13]. However, the reaction from $B$ could differ according to whether and how clearly A remembers B. B's memory on A might have diminished but only to a degree it could revive or to a completely oblivion. In a lesser oblivious instance B would come back saying "Yeah! Now I remember." Of course, A could be a stranger to B but A could have mistaken B. A more complex case is when A thought it was impossible for B not to know A, suspecting B was pretending in order to escape from A, say, B being indebted to A. Before taking actions, agents contemplate diverse epistemic contents though the visible behaviors resulting from such reasoning may be visually embodied into the same small set of scenes, which is ostensibly identical to that without the differentiated episteme [13].

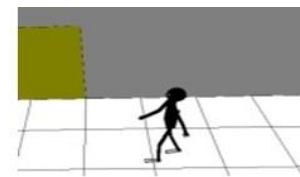

1 walking

3 handshaking

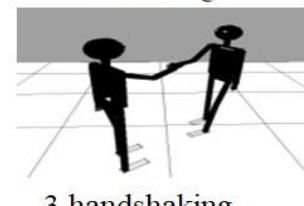

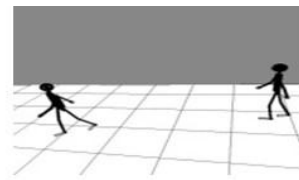

2 encounter

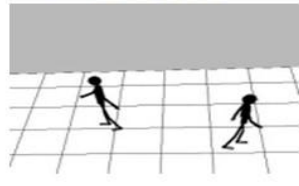

4 departing
Fig. 3. Several scenes from a story flow branched due to interperson epistemic discrepancies

As a second example, the interweaved situation illustrated in Fig. 4 involves initially-independent or derived events of a party, a burglary, a street check and a food poisoning. These events could be dynamically coupled with each other along their execution time possibly into lengthy implicative situations.

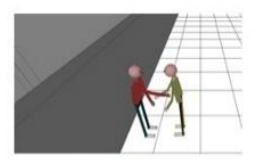

1 colluding

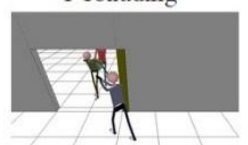

3 break-in
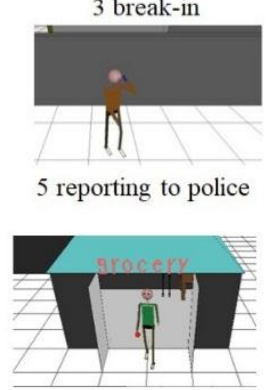

7 buying foodstuff

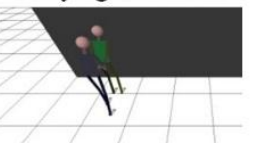

9 apprehended

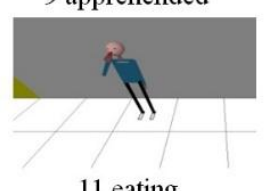

11 eating

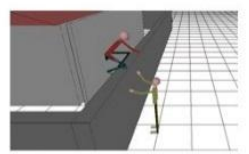

2 clearing fence

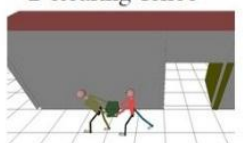

4 carrying
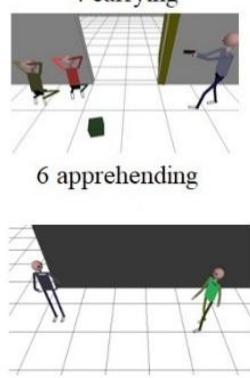

8 questioned

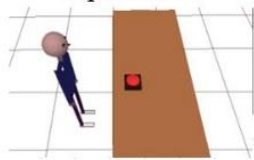

10 cooking

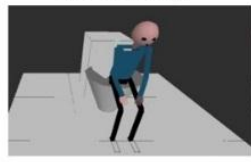

12 upset stomach
Fig. 4. One of the countless scene sequences to represent a situation interweaving two events

They are conducted by their associated agents through diverse actions including phone, talk, Fwalk, grasp, push and climb. Not all agents are shown in the screenshots but only those playing their respective parts of two burglars (B1, B2), hostess $(\mathrm{H})$, the victim and H's husband $(\mathrm{V})$, a policeman $(\mathrm{P})$, and 
party-goer (G). Still their behavior may well be affected by other agents behind the scene such as party participants, accomplices, fellow policemen, even passers-by. The scenes show those agents associated with collusion and theft followed by a police arrest, and a party. We first describe the story flow in terms of the major events in an omniscient view or independent of epistemology [5]: Burglary suspects are still at large; $\mathrm{H}$ had asked $\mathrm{V}$ on his way home to buy some foodstuff for cooking in preparation for the party; $\mathrm{V}$ was stopped by $\mathrm{P}$ on his way back home. These events and scenes could be sequenced into countless possible flows of situation, depending first on the conditions of agents and the rest of the background world as well as their interaction timing. One possible sequence might result from a condition where B1 or B2 was not detected in their burglary by $\mathrm{H}$ or any person, or $\mathrm{P}$ was too far away to get to the scene before B1 and B2 ran away when receiving H's burglary report. This failure to apprehend the burglars might send shockwaves to mistaken identity of an innocent husband in a street check to recapture them, to rotten foodstuff being carried by the victim, and all the way to food poisoning on party guests. Incidentally, these independent events (and their associated agents) are dynamically and often coincidentally coupled and affect each other regardless of non-deterministic timing of their couplings, collectively generating numerously diverse situations [14].

On top of these omnisciently-viewed situations of the reality, the epistemic discrepancies as elaborated for the previous example are applicable to this example situation as well. A misconception of B's on H's idea on B's location, for example, that $\mathrm{H}$ is not aware of B being in H's premise, could lead B's to behave less carefully than would otherwise, boosting the chance of being detected. Concerning the unfortunate party guests, if the questioning policeman had been prudent enough (or V's looks differ obviously enough from B's description) not to arrest $\mathrm{V}$, the foodstuff would not have gone bad with no chance of food poisoning in the first place. Likewise, the cook could have been sensitive enough to suspect the foodstuff beforehand. These epistemic variations could lead to numerous different orderings. This lengthy causality chain illustrates a cause event is not confined to an event that affects currently and directly, but could be any event, epistemically chained, which might have occurred earlier or whose consequences might have propagated from causality links away. In fact, the discrepancy between fact and belief and inter-agent epistemic discrepancy are general enough to affect any moment of agents' behaviors. The episteme such as typical or planned situations allows agents to proactively prepare for the future.

\section{DISCUSSION AND CONCLUSION}

We have developed a virtual-world simulation method where each agent has its own episteme to achieve a maximal variability and authenticity of situations. Unlike most of the serious games and conventional narrative systems, we indiscriminately model those agents to be autonomous humans regardless of their roles. That is, they have their own beliefs enabling them to behave autonomously and independently from each other. This epistemic differentiation itself provides a major source of additional situation variability compared to the omniscient-view or protagonist-centered situations of those conventional narrative systems. Each agent is designed to be incessantly update their respective episteme according to everchanging background-world conditions to adapt their behavior proactively and reactively. Such episteme cannot be perfect due to incomplete information and partial observation, leading to misconception and epistemic discrepancies, between fact and belief and inter-agent, further deepening the situation variability. With epistemic multiplicity for autonomous and independent agents our simulation method affords the highest diversity and fidelity of pedagogical situations involving multiple agents. The authoring scalability problem that is likely to worsen with our simulation scheme is resolved by the dynamic event coupling scheme and a real-time animation method. Our simulation method enables diverse situations to coherently develop by dynamically coupling the schematic events according to the association rules against their associated agents' back-ground world. We briefly described how dynamic event coupling scheme and a real-time animation method together resolves authoring scalability problem compared to monolithically-authored conventional simulation systems. We demonstrated the feasibility of our simulation method by implementing example situations. Our simulation method can methodically realize narrative variability that conventional simulation methods ardently pursue and achieve the highest diversity and fidelity of reality for multi-agent situations. Further research includes affective natures of humans. The affective reaction is reified as a result of a lengthy, complicated mental process based on episteme and values among other mental elements. Those elements encompass sensational, rational and emotional ones.

\section{ACKNOWLEDGEMENTS}

This research was supported by Basic Science Research Program through the National Research Foundation of Korea(NRF) funded by the Ministry of Education(NRF2020R1I1A3069589).

\section{REFERENCES}

[1] Marsella S, Johnson W, LaBore C. Interactive pedagogical drama for health interventions. Proc. of AIED 2003, Australia. 2003.

[2] Zook A, et al. Automated scenario generation: toward tailored and optimized military training in virtual environments. Proc. of the International Conference on the Foundations of Digital Games, ACM. 2012.

[3] Rui F, et al. Emergent stories facilitated, story. Springer, Berlin, Heidelberg. 2008:218-229.

[4] Bailenson J, et al. The use of immersive virtual reality in the learning sciences: digital transformations of teachers, students, and social context. The Journal of the Learning Sciences. 2008:102-141.

[5] Hong S, et al. Simulation of concurring multi-agent events with individualized episteme to maximize variability of pedagogical situations for immersed 
International Journal of Engineering Research and Technology. ISSN 0974-3154, Volume 13, Number 8 (2020), pp. 2090-2095

(C) International Research Publication House. https://dx.doi.org/10.37624/IJERT/13.8.2020.2090-2095

intelligent tutoring system. Proc. Of ICERI, Seville, Spain. 2018.

[6] Park J. Implementation of an Agent-Centric of Events as Objects of Pedagogical Experiences in Virtual World, Int'l Journal of Contents. 2016:12(1):25-43

[7] Choi J, Park J. An Effective implementation of agent's complex actions by reusing primitive motions. Proc. of Simultech, Vienna, Austria. 2014.

[8] Charles F, et al. Planning formalisms and authoring in interactive storytelling. Proc. of International Conference on Technologies for Interactive Digital Storytelling and Entertainment. 2003.

[9] Porteous J, Cavazza M, Charles F. Applying planning to interactive storytelling: Narrative control using state constraints. ACM Trans. on Intelligent Systems and Technology. 2010:1(2).
[10] Kettunen J. Constructing Identities In San Andreas: Characterizing the protagonists in Grand Theft Auto V. University of Jyvaskyla, Aug. 2015.

[11] Choi J, Park J. A Student-directed Immersive Intelligent Tutoring System for Language Learning. Proc. of ITS, Zagreb, Croatia, 2016.

[12] Cavazza M, Charles F, Mead S. Emergent situations in interactive storytelling. Proc. of ACM Symposium on Applied Computing (ACM-SAC), Madrid, Spain. 2002.

[13] Park J, Choi J. A Situation Simulation Method for Virtual Situations Through Seamless Integration of Independent Events via Autonomous and Independent Agents. Int'1 Journal of Contents. 2018:14(3). 